\title{
RESPUESTA SÍSMICA EN EL LAGO DE TEXCOCO. RESULTADOS A PARTIR DE REGISTROS DE MOVIMIENTOS FUERTES
}

\author{
Hortencia Flores Estrella $^{(1)}$, Martín Cárdenas Soto ${ }^{(2)}$ y Cinna Lomnitz $^{(3)}$
}

\begin{abstract}
RESUMEN
En 1995 se instaló un arreglo acelerométrico constituido por seis estaciones en superficie, que formaron dos triángulos con aperturas de 30 y $400 \mathrm{~m}$ y un arreglo vertical con instrumentos en cinco profundidades, que alcanzaron los $40 \mathrm{~m}$. La base de datos de este arreglo consta de ocho eventos de movimientos fuertes $(5.9<\mathrm{M}<7.9)$ que analizamos para obtener la respuesta sísmica, el modelo de velocidades y el espectro de respuesta de sitio. Los resultados de este estudio muestran que la respuesta de sitio está caracterizada por la frecuencia fundamental en $0.37 \mathrm{~Hz}$. El análisis de los registros en pozo permitió obtener un modelo de velocidades que está constituido por dos capas, con factores de atenuación Q distintos para cada una; la amplificación dinámica, debida a las propiedades físicas de las arcillas lacustres, domina la respuesta de sitio. Finalmente, el espectro de respuesta muestra que las seudoaceleraciones máximas en el sitio se encuentran en dos intervalos de periodo: entre 0 y $1 \mathrm{~s}$ y entre 2 y $3 \mathrm{~s}$, con ordenadas espectrales de $0.6 \mathrm{~g}$ y $0.74 \mathrm{~g}$, respectivamente.
\end{abstract}

\begin{abstract}
In 1995 an accelerometer array was installed, it consisted on six surface stations which formed two triangles with distances of 30 and $400 \mathrm{~m}$ between them, and a vertical array with instruments in five depths. The database is formed by eight strong motion records $(5.9<\mathrm{M}<7.9)$, which were analyzed to estimate the seismic response, to obtain a velocity model for the clay layer and to calculate the response site spectra. The site response is characterized by the fundamental frequency on $0.37 \mathrm{~Hz}$. and an amplification factor around. The shear velocity model was obtained from the analysis to the seismic records from the borehole; this model consists on two layers with a Q factor estimation for each of them. The transfer function for this model is dominated by the dynamic amplification, due to the lacustrine clay layer and its physical properties, and it is close to the empirical transfer function obtained from spectral ratios. The response spectra estimation shows two intervals with maximum seudoacceleration: between 0 and $1 \mathrm{sec}$ and between 2 and $3 \mathrm{sec}$, with spectral ordinates of 0.6 and $0.74 \mathrm{~g}$, respectively.
\end{abstract}

\section{INTRODUCCIÓN}

La Ciudad de México, al ser una de las urbes más pobladas del mundo y el centro político del país, es el centro de atención de estudios enfocados a conocer la respuesta sísmica del terreno a fin de reducir el riesgo ante sismos de gran magnitud. Por ello el movimiento del terreno durante sismos fuertes, es un tema que ha sido estudiado por distintos grupos de investigación, desde enfoques tanto teóricos como

Artículo recibido el 13 de marzo de 2009 y aprobado para su publicación el 23 de diciembre de 2009. Se aceptarán comentarios y/o discusiones hasta cinco meses después de su publicación

${ }^{1}$ Departamento de Sismología, Instituto de Geofísica, UNAM.

${ }^{2}$ Departamento de Geofísica, División de Ingeniería en Ciencias de la Tierra, Facultad de Ingeniería, UNAM

${ }^{3}$ Departamento de Sismología, Instituto de Geofísica, UNAM 
prácticos. La importancia de este tema radica en que los efectos de sitio en algunas zonas de la ciudad ocasionan que la respuesta sísmica esté caracterizada por una amplificación significativa del movimiento (generada de manera local y regional) y por una larga duración del mismo.

Los sismos de Michoacán de septiembre de 1985 causaron uno de los peores desastres en la ciudad (350 edificios dañados o colapsados y una cantidad no consensuada de pérdidas humanas), principalmente en las zonas ubicadas sobre el lecho del Lago de México, sobre el cual la ciudad creció después de que éste se desecó. A partir de estos eventos, los efectos de sitio en la zona de lago de la Cuenca de México cobraron mayor interés de distintas disciplinas debido a las consecuencias destructivas de éstos. En primer instancia, el análisis de los registros de estos eventos reportó que el efecto de sitio en la zona de lago de la ciudad, estuvo caracterizado por una amplificación del movimiento por un factor de 40 veces respecto de los sitios en zona de lomas (Singh et al., 1988); este fenómeno se explicó como resultado del contraste de impedancias entre el suelo arcilloso y los estratos más competentes. Además, se observó que la amplificación del movimiento del terreno en la Ciudad de México no es exclusiva de los sitios en la zona de lago. Gracias al análisis de los registros de los sismos de 1985 se encontró que en la zona de lomas de la ciudad existía una amplificación del movimiento en frecuencias entre 0.2 y 2 Hz (Ordaz y Singh, 1992; Singh et al., 1995; Pacheco y Singh, 1995). Cárdenas-Soto et al. (1997) delimitaron la zona en la cual se observa esta amplificación regional y la asociaron con una estructura compleja debajo de la parte sur y centro de México, como podría ser una discontinuidad en el límite sur del Cinturón Volcánico Transmexicano, CVT (Chávez-García et al., 1995; Chávez-García y Salazar, 2002)

En las estaciones localizadas en los suelos de origen lacustre, Beck y Hall (1986) identificaron una larga duración del movimiento en los registros de los sismos de 1985; este fenómeno está caracterizado por aparecer como una sucesión de paquetes de ondas armónicas (Singh y Ordaz, 1993) y ha sido extensivamente documentado por diversos autores (ibid; Beck y Hall, 1986; Lomnitz, 1988, 1990; Alvarez et al., 1995; Chávez-García et al., 1995; Barker et al., 1996; Iida, 1999; Roullé y Chávez-García, 2005; Cárdenas-Soto y Chávez-García, 2007). Sobre el origen de este fenómeno se han propuesto explicaciones que involucran efectos de sitio 2D y 3D, perturbaciones gravitacionales de las ondas superficiales o generación de ondas superficiales en los límites de la cuenca. Chávez-García y Bard (1993, 1994) analizaron y discutieron si estos factores podrían ser las causas de esta duración. Algunos resultados a partir del análisis de sismos fuertes (Chávez-García et al., 1995; Roullé y Chávez-García, 2005; CárdenasSoto y Chávez-García, 2007) apuntan a que la larga duración del movimiento, en la zona de lago de la Ciudad de México, es debida a una interacción de ondas guiadas por la estructura profunda de la cuenca (entre $1 \mathrm{y} 4 \mathrm{~km}$ ) con la resonancia 1D de las capas superficiales; o bien, que existe un acoplamiento entre ondas $\mathrm{P}$ y Rayleigh, que aparece como pulsos monocromáticos que son debidos a las condiciones de velocidad de la parte somera de la Cuenca de México (Lomnitz et al., 1999). En la actualidad no existe un consenso sobre el origen y la causa de este fenómeno, cuyo entendimiento es primordial para reducir el riesgo sísmico de la ciudad, pues dado que arribos sucesivos de paquetes energéticos de ondas imponen cargas laterales adicionales a las obras civiles.

La evaluación de los efectos de sitio en la cuenca ha sido realizada en su mayor parte en la zona urbana; sin embargo, recientemente ha surgido una serie de estudios que han cuestionado el llamado "movimiento de campo libre" en zonas urbanas. En efecto, muchas de las mediciones han sido en la vecindad de edificios, y es bien sabido que éstos, al interactuar con el movimiento del suelo, producen el llamado efecto de Interacción Suelo-Estructura. El análisis de los registros de algunos edificios instrumentados muestra que la existencia de efectos de interacción suelo-estructura puede modificar la respuesta sísmica del terreno (Chávez-García y Cárdenas-Soto, 2002).

Un fenómeno adicional que debe considerarse en la evaluación de la respuesta sísmica, en la zona urbana de la cuenca, es la compactación de las arcillas por el hundimiento de la Ciudad de México, 
resultado del bombeo del agua del subsuelo para el consumo local. Ovando et al. (2007) mostraron que este fenómeno ocasiona una consolidación del terreno, que a su vez genera un aumento en los esfuerzos de cizalla efectivos que modifican tanto el módulo de cortante, como la velocidad de propagación de ondas $\mathrm{S}$ de los depósitos lacustres de la cuenca. Estos autores (ibid) proponen que esto deberá verse reflejado en una revisión constante de los mapas de zonificación y de los espectros de diseño asociados.

Los estudios mencionados han permitido contar con una gran cantidad de información en torno a la respuesta sísmica de la Ciudad de México; sin embargo, el crecimiento de esta urbe se ha extendido hacia los lechos de los antiguos lagos como son los de Chalco, de Xochimilco y de Texcoco; prueba de ello es el desarrollo y el crecimiento en número de habitantes de algunos municipios del Estado de México y de algunas delegaciones del Distrito Federal, D. F. (por ejemplo: Chalco, Ecatepec, Texcoco, Xochimilco, ver tabla 1); además, se han dado esfuerzos gubernamentales para la construcción de infraestructura de servicios, tales como la propuesta alternativa del aeropuerto de la ciudad, sobre el lecho de lo que era el Lago de Texcoco, al NE de la Ciudad de México.

Tabla 1. Crecimiento en número de habitantes de algunos municipios o delegaciones en la zona de lago de la Cuenca de México, entre 1995 y 2005 (fuente INEGI).

\begin{tabular}{ccc}
\hline Municipio o delegación & No. de habitantes (1995) & No. de habitantes (2005) \\
\hline Chalco & 175,821 & 257,405 \\
Ecatepec & $1,457,124$ & $1,688,258$ \\
Tecamac & 148,432 & 270,574 \\
Texcoco & 173,106 & 209,308 \\
Xochimilco & 332,314 & 404,458 \\
\hline
\end{tabular}

En un proyecto conjuntos de los Institutos de Ingeniería y de Geofísica de la UNAM y como un esfuerzo por conocer la respuesta sísmica en arcillas vírgenes de la Cuenca de México, en 1995 se instaló un arreglo instrumental sobre las arcillas del Lago de Texcoco, dentro de la Reserva Natural a cargo de la Comisión Nacional del Agua (CNA), al NE de la Ciudad de México (arreglo TXCL en la figura 1). El objetivo del arreglo instrumental Texcoco, TXCL, fue el de monitorear la propagación de ondas superficiales coherentes y enriquecer el conocimiento sobre el campo de ondas que se propaga ante la incidencia de movimientos fuertes (Stephenson y Lomnitz, 2005).

En este estudio analizamos la base de datos de registros de movimientos fuertes del arreglo TXCL con el propósito de caracterizar la respuesta de sitio. Para ello empleamos las técnicas de cocientes espectrales H/V (Lermo y Chávez-García, 1993) y de cocientes espectrales estándar, SSR por sus siglas en inglés (Borcherdt, 1970) y realizamos una evaluación de la frecuencia fundamental y de la amplificación dinámica de los estratos de arcilla en la región. Posteriormente, con el apoyo de los registros en el arreglo vertical estimamos un modelo de velocidad de ondas de corte a fin de reproducir las observaciones. Finalmente, utilizando los registros de movimientos fuertes proponemos un espectro de respuesta de sitio para la zona de estudio.

\section{ANTECEDENTES GEOLÓGICOS Y GEOTÉCNICOS EN EL LAGO DE TEXCOCO}

El lecho del Lago de Texcoco está constituido por arcillas de origen lacustre, que son las mismas que se extienden en la zona de lago de la Ciudad de México; además, es una región cuya respuesta sísmica no se ha caracterizado completamente (Reglamento de Construcciones del Distrito Federal, RCDF, 2004; Mayoral et al., 2008). En los tres primeros metros de profundidad las arcillas presentan grietas de desecación poco profundas, que se extienden por varios metros de manera horizontal (Mayoral et al., 2008). En los siguientes 25 metros se encuentran capas de arcillas intercaladas con lentes de limos 
arenosos o de arena limosa, hasta llegar a la llamada capa dura que está formada por limos arenosos, arenas y limos con un espesor de hasta 3.5 metros; debajo de ésta existen más arcillas hasta los $42 \mathrm{~m}$ de profundidad, donde se comienzan a encontrar suelos profundos de limos consolidados y arenas limosas (Pérez-Cruz, 1988; Stephenson y Lomnitz, 2005; Mayoral et al. 2008).

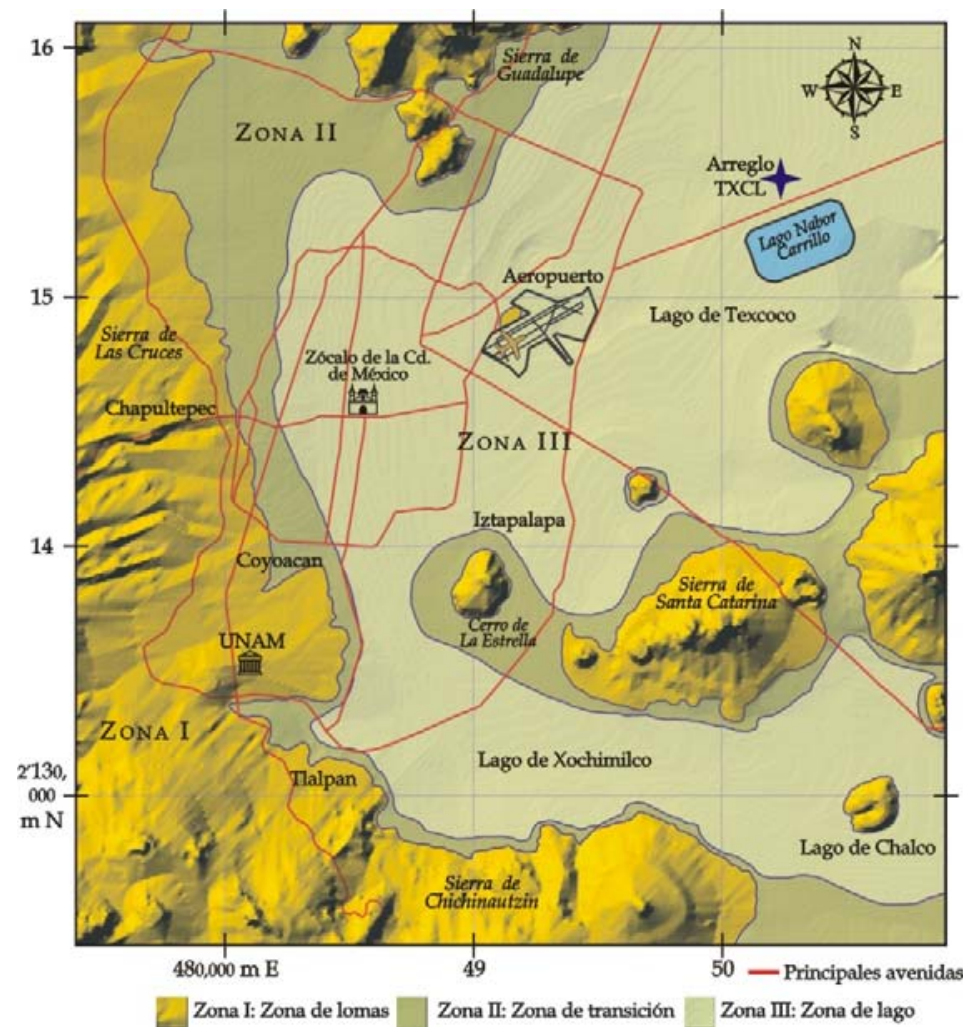

Figura 1. Zonificación geotécnica de la Cuenca de México y la localización del arreglo TXCL al NE de la ciudad (adaptada de Flores-Estrella et al., 2007)

Las arcillas de origen lacustre de los primeros metros de la columna estratigráfica se caracterizan por tener un alto índice de plasticidad, por no presentar una reducción del módulo de cortante para deformaciones de hasta el 1\% (Mayoral et al., 2008) y por ser altamente compresibles, lo que implica que tengan una respuesta lineal ante los esfuerzos a los que se someten durante un evento sísmico. El módulo de Poisson de estos materiales es cercano a 0.49, tienen una densidad de $1,100 \mathrm{~kg} / \mathrm{cm}^{3}$ (Stephenson y Lomnitz, 2005; Stephenson et al., 2005) y una salinidad muy alta que puede provocar problemas en la instrumentación a corto y mediano plazo. El contenido de agua de los depósitos es descrito por Stephenson y Lomnitz (2005) a partir del análisis de un núcleo de $120 \mathrm{~m}$; en los primero $28 \mathrm{~m}$ se tiene un contenido de agua del $300 \%$ que disminuye al $25 \%$ entre los 100 y $140 \mathrm{~m}$ de profundidad y se asume que debajo de los $160 \mathrm{~m}$ de profundidad el contenido de agua disminuye al 25\%, lo que sugiere la existencia de una capa más rígida.

En el área de estudio se han realizado una serie de estudios geológicos, geofísicos y geotécnicos con el objetivo de conocer la respuesta sísmica de sitio. Ramos-Martínez et al. (1997) realizaron un estudio de refracción, a partir del cual encontraron un modelo de velocidades de dos capas (de 18 y de $15 \mathrm{~m}$ de espesor) sobre un semi-espacio y una estimación del factor de atenuación Qs de 42. Posteriormente, Aguirre et al. (2001) y Flores-Estrella (2004) realizaron análisis de microtremores registrados en la zona, 
para caracterizar la respuesta sísmica del terreno y obtener una estimación del modelo de velocidades; sus respectivos modelos son similares entre sí y varían en cuanto a la profundidad a la que se localiza la capa dura y al valor de la velocidad de ésta; los resultados de Aguirre et al. (2001) llegaron a definir las velocidades hasta una profundidad de 60 m, mientras que Flores-Estrella (2004) llegó a 39 m.

Stephenson y Lomnitz (2005) realizaron estudios de penetrometría de cono sísmico (SCPT), con el propósito de definir un modelo de velocidades; este modelo está compuesto por nueve capas y considera dos inversiones de velocidades: la primera debajo de la capa dura a los $29 \mathrm{~m}$ de profundidad y la segunda debajo de los $35 \mathrm{~m}$ de profundidad; además sugieren que a los $39 \mathrm{~m}$ se marca un cambio en la litología, que está caracterizado por el comportamiento del material. Los resultados de estos autores concuerdan con las estimaciones de velocidades realizadas por estudios previos (Ramos-Martínez et al., 1997; Aguirre et al., 2001; Flores-Estrella, 2004) hasta la profundidad de la capa dura (29 m).

Recientemente, Mayoral et al. (2008) estudiaron las propiedades dinámicas de las arcillas de la zona de Texcoco Sosa, que está situada a $9 \mathrm{~km}$ al norte del arreglo TXCL y que en el RCDF (2004) está considerado como zona de transición. Mayoral et al. (2008) determinan una estratigrafía muy similar a la descrita previamente por Pérez-Cruz (1988) y por Stephenson y Lomnitz (2005); además, presentan la estimación de un espectro de respuesta para la zona a partir de los registros de eventos sísmicos en la estación TXSO. Sus resultados sugieren que se requieren más estudios sobre la respuesta sísmica del área, para lograr que se tenga una caracterización completa que pueda ser considerada por el RCDF, con fines de reducción del riesgo sísmico en la zona de lago de la Cuenca de México.

\section{DATOS}

El arreglo instrumental TXCL funcionó de 1995 a 2004; en la figura 3 se muestra un esquema de éste, los puntos negros indican la posición de los acelerógrafos, tanto en superficie como en el arreglo vertical. El arreglo TXCL consistió de seis acelerográfos en superficie formando dos arreglos triangulares con aberturas de aproximadamente 40 y $300 \mathrm{~m}$; en un vértice del arreglo de $300 \mathrm{~m}$ (tx5a en la figura 2) se instalaron sensores en las profundidades de 2, 10, 20, 30 y $40 \mathrm{~m}$. En la figura 3 se obvia la posición del sensor en dos metros de profundidad, pues la escala no permite verlo claramente. En las estaciones txs1 y txs2 los instrumentos que se usaron fueron acelerográfos ETNA, de Kinemetrics y para las estaciones t4s1, t4s2, t4s3 y txs5a se usaron instrumentos K2. En ambos tipos de instrumentos la velocidad de muestreo fue de 100 muestras por segundo.

De los registros de movimiento fuertes obtenidos durante el periodo de operación del arreglo TXCL, seleccionamos ocho eventos con magnitud mayor a seis (tabla 2) registrados en al menos dos estaciones y cuyo registro estuviera completo en los tres componentes del movimiento. Los eventos seis y siete de esta tabla están registrados en los instrumentos del arreglo vertical, el primero en las profundidades $0,2,10,2030$ y $40 \mathrm{~m}$ y el segundo en las profundidades $0,2,10$ y $20 \mathrm{~m}$.

En la figura 3 se muestran los registros del arreglo vertical en el componente NS para el evento número 6 (ver tabla 2). En esta figura se puede ver cómo la amplitud en la parte intensa del registro (entre 25 y $55 \mathrm{~s}$ de tiempo) disminuye con la profundidad, la amplitud en el registro en superficie es hasta cuatro veces mayor con respecto al registro obtenido en los $40 \mathrm{~m}$ de profundidad; además se tiene el efecto debido a la amplificación por superficie libre. En el registro del instrumento en la profundidad de $2 \mathrm{~m}$, la amplitud de la coda es similar a aquella de la parte intensa, pues el arribo de ondas de distintas direcciones juega un papel fundamental en este fenómeno. Resultados similares se observan en los registros del componente EW, no mostrado por cuestiones de brevedad. 


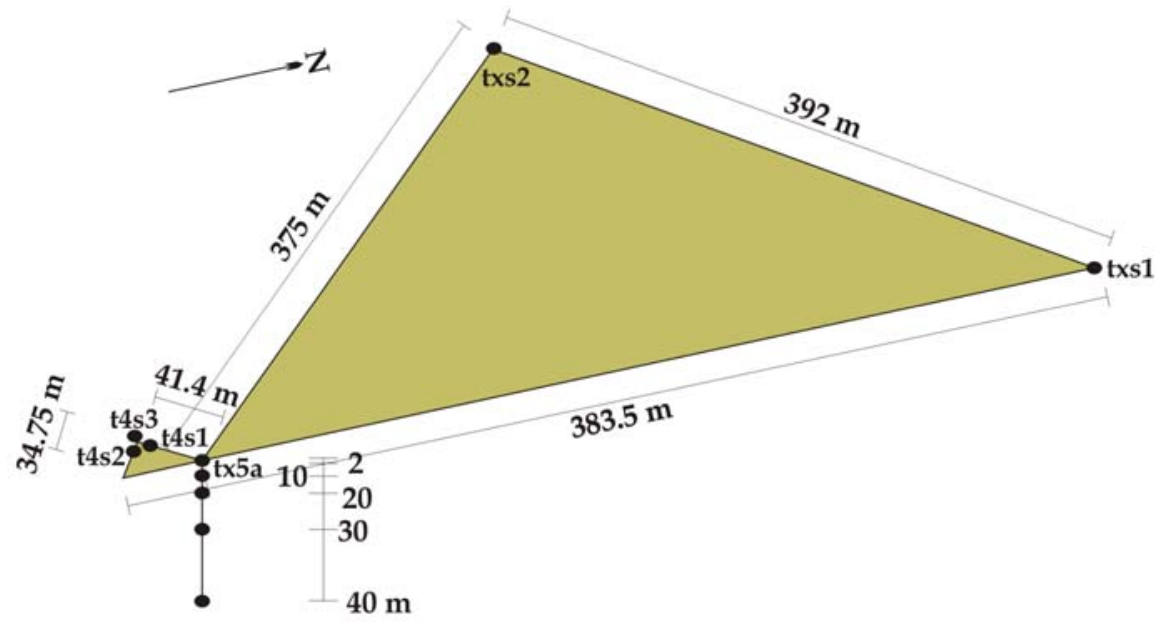

Figura 2. Esquema del arreglo TXCL. Los puntos negros indican la posición de los instrumentos en superficie (seis estaciones) y en el arreglo vertical a profundidades de 2, 10, 20, 30 y $40 \mathrm{~m}$.

Tabla 2. Localización y características de los eventos sísmicos seleccionados.

\begin{tabular}{cccccccc}
\hline & $\begin{array}{c}\text { Evento } \\
\text { (dd.mm.aaaa) }\end{array}$ & $\mathrm{M}$ & Lat $^{\circ} \mathrm{N}$ & Long $^{\circ} \mathrm{W}$ & $\begin{array}{c}\text { Profundidad } \\
(\mathrm{km})\end{array}$ & Baz & $\begin{array}{c}\text { Distancia } \\
\text { epicentral }(\mathrm{km})\end{array}$ \\
\hline 1 & 20.04 .1998 & 5.9 & 18.37 & 101.21 & 66.0 & 242.551 & 266.202 \\
2 & 15.06 .1999 & 6.4 & 18.18 & 97.51 & 69.0 & 133.02 & 240.05 \\
3 & 21.06 .1999 & 6.0 & 17.99 & 101.72 & 54.0 & 240.575 & 333.784 \\
4 & 30.09 .1999 & 6.5 & 15.95 & 97.03 & 16.0 & 151.975 & 442.612 \\
5 & 08.10 .2001 & 6.1 & 16.94 & 100.14 & 4.0 & 203.781 & 308.108 \\
6 & 10.11 .2001 & 6.0 & 15.96 & 98.17 & 9.0 & 167.55 & 399.77 \\
7 & 22.01 .2003 & 7.6 & 18.60 & 104.22 & 9.0 & 260.751 & 560.890 \\
8 & 01.01 .2004 & 6.3 & 17.34 & 101.42 & 6.0 & 227.722 & 351.317 \\
\hline
\end{tabular}

\section{ESTIMACIÓN DE LA RESPUESTA DE SITIO}

Los dos métodos más utilizados para la estimación de la respuesta de sitio son los cocientes espectrales H/V en una misma estación de registro (Lermo y Chávez-García, 1993) y los cocientes espectrales estándar o respecto de una estación de referencia, SSR por sus siglas en inglés (Standar Spectral Ratios) (Borcherdt, 1970); en ambos casos se obtiene una función de transferencia empírica, definida por la frecuencia fundamental y el nivel de amplificación. A fin de obtener la respuesta de sitio en la zona del arreglo TXCL, se consideraron los registros de los componentes Z, NS y EW; el análisis se realizó para ventanas de $40 \mathrm{~s}$ de duración a partir del inicio del movimiento intenso y se usó el programa DEGTRA A4 (Ordaz y Montoya, 2005). A cada ventana de registro se le aplicó un taper del 5\% de la longitud total de la ventana y posteriormente se calcularon los espectros de amplitud de Fourier, los cuales fueron suavizados mediante una ventana que conserva la energía en frecuencias altas. En la estimación de los cocientes $\mathrm{H} / \mathrm{V}$ se consideraron los registros de las estaciones en superficie de todos los sismos de la tabla 2. Para la evaluación de los cocientes SSR se consideraron los registros de los sismos dos, tres y cuatro de la tabla 2 en las estaciones en superficie del arreglo TXCL y en la estación Estanzuela, ESTS, situada aproximadamente a $9 \mathrm{~km}$ al oeste del Lago de Texcoco. Además, para los cocientes SSR también se consideraron los componentes de movimiento horizontal rotados según la dirección epicentral. 


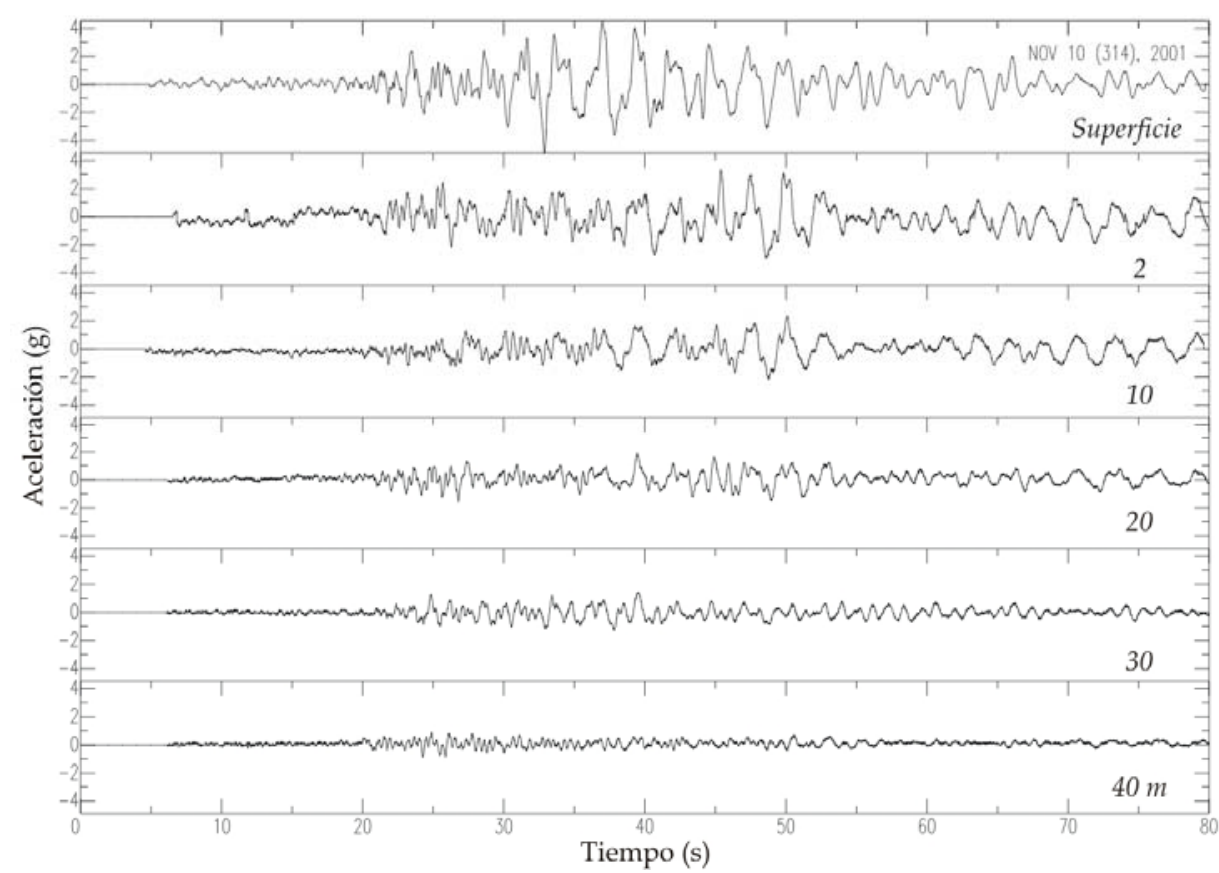

Figura 3. Registros de la componente NS del evento número 6 (ver tabla 2) en las estaciones del arreglo vertical, en las profundidades de 0, 2, 10, 20, 30 y $40 \mathrm{~m}$.

En la figura 4 se muestran los resultados de los cocientes espectrales para las estimaciones $\mathrm{H} / \mathrm{V}$, para los que se consideraron los componentes NS, EW y vertical. Se puede ver que las formas espectrales son similares entre sí para ambas direcciones del movimiento. En esta figura se puede ver una frecuencia fundamental en $0.37 \mathrm{~Hz}$, con un factor de amplificación por un factor de 7.6 y tres armónicos superiores en $0.1,1.5$ y $2.5 \mathrm{~Hz}$.

En la figura 5 se muestran los cocientes espectrales estándar para los componentes NS y EW, en la parte izquierda y para los componentes radial y transversal del movimiento, en la parte derecha; las líneas delgadas corresponden con cada uno de los cocientes y la líneas más gruesa indica el promedio total de éstos.

En la figura 5 izquierda se puede ver que, para el caso de los cocientes con los componentes NS y EW, hay un pico principal en $0.37 \mathrm{~Hz}$, con una amplificación relativa por un factor de 16 y tres armónicos en 1.1, 1.4 y $1.7 \mathrm{~Hz}$. Por otro lado, en la parte derecha se puede ver que para los cocientes espectrales de los componentes rotados hacia las direcciones radial y transversal de cada evento, la frecuencia fundamental está en $0.37 \mathrm{~Hz}$, con un factor de amplificación relativa de 15; también se pueden ver tres armónicos superiores en las frecuencias de 1.1, 1.4 y $1.7 \mathrm{~Hz}$.

La respuesta de sitio en el Lago de Texcoco está definida por una frecuencia fundamental en $0.37 \mathrm{~Hz}$ y valores de amplificación que varían entre un factor de 7 y de 17. Estos factores parecen estar subestimados, lo cual se ha visto que es normal para los cocientes espectrales $\mathrm{H} / \mathrm{V}$; no así para los cocientes espectrales estándar, con los que se han encontrado factores de amplificación relativa cercanos a 50 veces (Singh et al., 1988 y 1995) 


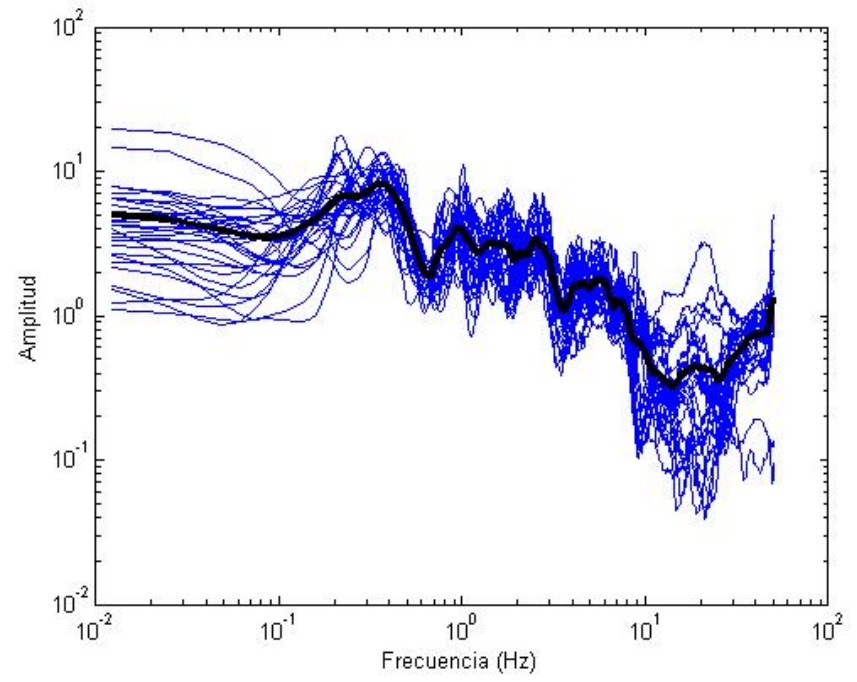

Figura 4. Cocientes espectrales H/V en el arreglo TXCL.
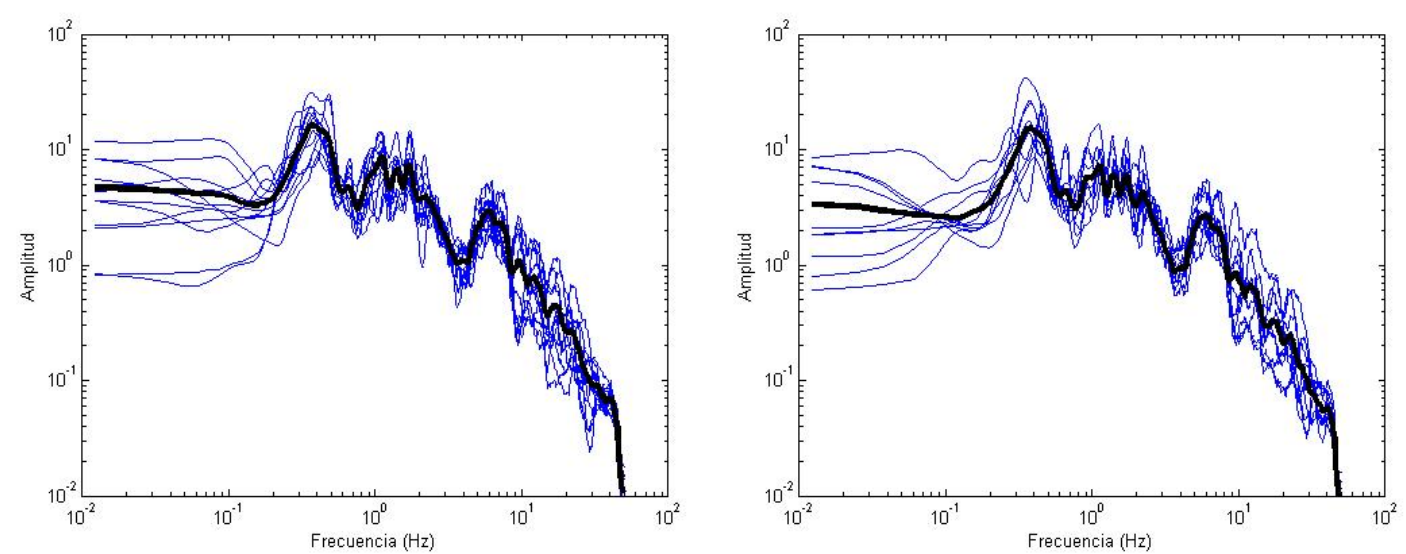

Figura 5. Cocientes espectrales estándar, respecto a la estación ESTS. En la parte izquierda los cocientes que consideran los componentes NS y EW y en la parte derecha los que consideran los componentes radial y transversal, respecto a la dirección epicentral.

\section{ESTIMACIÓN DEL MODELO DE VELOCIDAD DE ONDAS DE CORTE}

El análisis de los registros del arreglo vertical permite comprender y caracterizar la propagación del campo de ondas en profundidad, con el objetivo de obtener un modelo de velocidades de ondas de corte y una estimación del factor de calidad Q. Para ello utilizamos la técnica de interferometría sísmica, la cual proporciona una estimación de la función de Green mediante la correlación de ondas registradas entre pares de receptores (Sneider y Safak, 2006; Cárdenas-Soto y Chávez-García, 2007). En el caso de movimientos fuertes, realizamos la deconvolución del registro en la superficie del terreno con respecto de los registros en las distintas profundidades para los datos de los eventos seis y siete (tabla 2).

En la figura 6 se muestran las formas de onda deconvolucionadas de los componentes horizontales del registro del evento número siete (tabla 2). En esta figura se puede observar que en la superficie y en la 
deconvolución respecto al registro en $2 \mathrm{~m}$ de profundidad, se tiene una función delta; mientras que en la deconvolución respecto a las distintas profundidades aparece la parte anticausal y casual de la función de Green (la onda que sube y la que baja, respectivamente). Esta función representa el campo de onda que se propaga entre pares de receptores. En la figura 6 se observa que en ambas direcciones horizontales la forma y amplitud de la deconvolución es la misma, lo que indica que el modelo del subsuelo es simple y sin efectos de variaciones laterales.

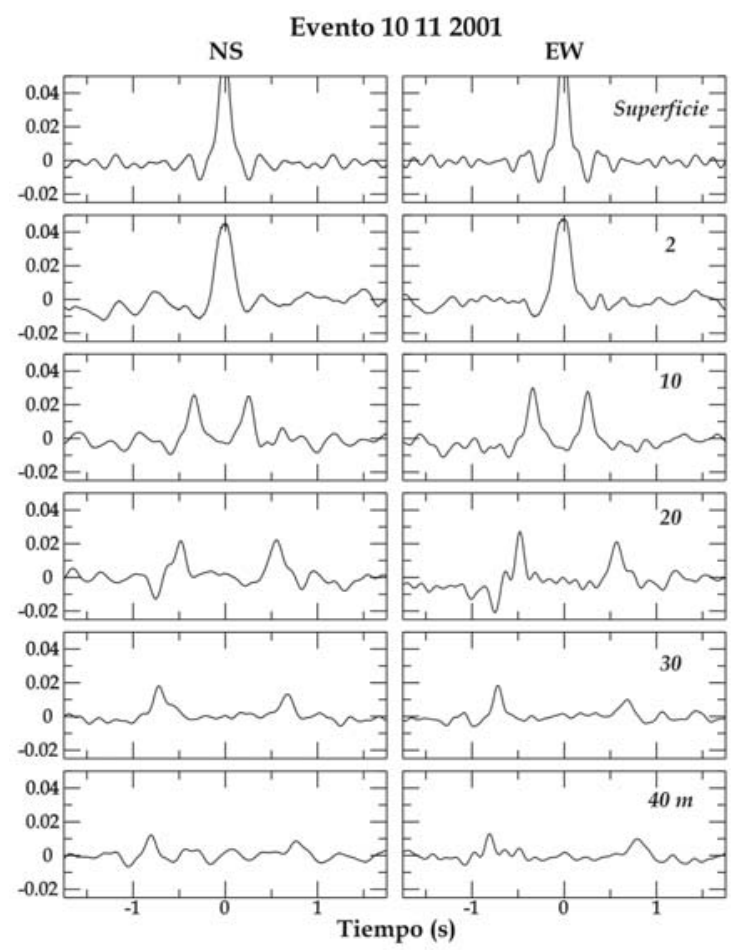

Figura 6. Formas de ondas deconvolucionadas de los registros en las distintas profundidades, respecto al registro en superficie; se consideraron los componentes NS y EW del evento número siete (ver tabla 2).

Los tiempos en que aparecen los máximos de las formas de onda de la decovolución y la distancia entre receptores fueron utilizados para construir curvas de tiempo de viaje que se muestran en la figura 7 . Debido a que los tiempos de viaje de las ondas ascendentes y descendentes son los mismos en ambas direcciones, los lugares geométricos correspondientes se sobreponen en la figura 6. Los tiempo de arribo de las ondas ascendentes definen claramente dos pendientes, que indican la velocidad de ondas de corte; entre la superficie y $20 \mathrm{~m}$ de profundidad esta velocidad es de $34 \mathrm{~m} / \mathrm{s}$ y entre 20 y $40 \mathrm{~m}$ de profundidad es de $79 \mathrm{~m} / \mathrm{s}$. En general, los tiempos de las ondas ascendentes pueden ser interpolados en las pendientes de estas dos velocidades.

Sneider y Safak (2006) obtienen el factor de calidad Q a partir de la deconvolución de los registros en las distintas profundidades respecto a la base de una estructura rígida. En este caso hemos deconvolucionado el registro a 40 metros de profundidad con respecto al resto, sin lograr obtener una estimación de Q. Esto se debe a que el registro a la profundidad de 40 metros aún se encuentra dentro de la capa arcillosa donde no existe una base rígida que atrape en forma considerable la propagación vertical de la energía. Sin embargo, en la figura 6 se observa que la amplitud de las deconvoluciones decrece con la profundidad. Siguiendo a Aki y Richards (1980), utilizamos la siguiente relación que expresa el decaimiento de la amplitud con la posición para una frecuencia fija y en la que z se refiere a la coordenada vertical: 
$A=A_{0} \exp (z \alpha)$

donde el factor $\alpha$ está relacionado al factor de calidad $Q$ mediante la siguiente relación:

$\alpha=\frac{-\omega}{2 c Q}$

donde $\omega$ es la frecuencia angular y $c$ es la velocidad de fase que consideramos como $c=0.92 \beta$, donde $\beta$ corresponde con la velocidad de ondas de corte.

En esta formulación se asume que la pérdida de amplitud con la distancia de deben a la atenuación intrínseca de los materiales. Para el caso de las arcillas del sitio TXCL, el fenómeno se debe a la disipación de energía durante la propagación vertical de las ondas a la frecuencia de resonancia del subsuelo. Así, dadas las dos pendientes de velocidad (figura 7), estimamos el valor de Q siguiendo la formulación anterior. En la tabla 3 se resumen los resultados obtenidos utilizando la técnica de interferometría sísmica en el arreglo vertical. Los valores de esa tabla son congruentes con los reportados en Stephenson y Lomnitz (2005), Flores-Estrella (2004) y Aguirre et al. (2001).

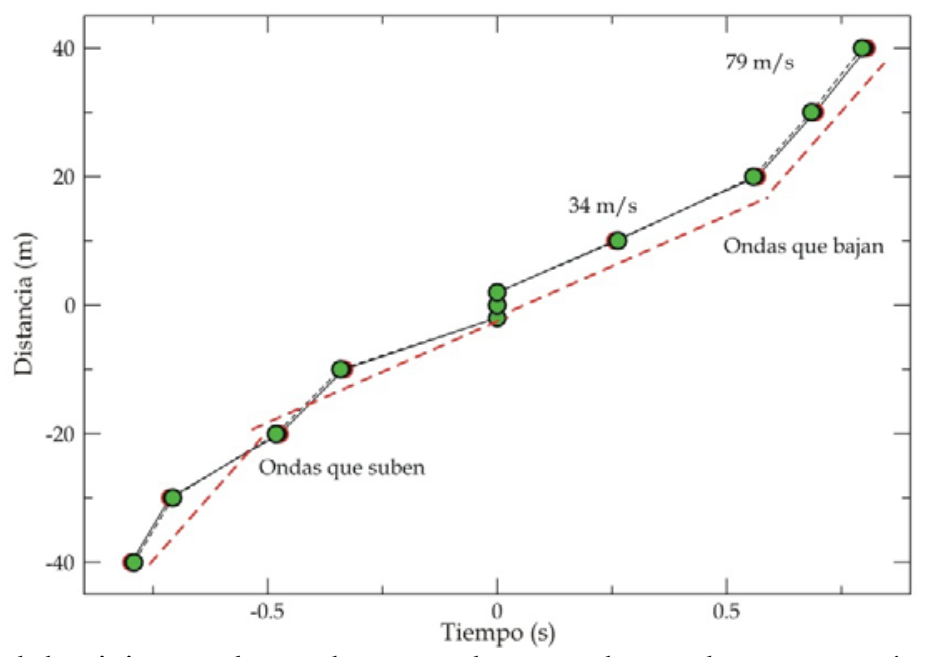

Figura 7. Velocidad de viaje para las ondas ascendentes y descendentes a través de la capa de arcilla lacustre de TXCL, a la izquierda y a la derecha de la gráfica, respectivamente.

Tabla 3. Modelo de velocidades de onda de corte, para el sitio TXCL

\begin{tabular}{cccc}
\hline Capa & $\begin{array}{c}\text { Profundidad } \\
(\mathbf{m})\end{array}$ & $\begin{array}{c}\boldsymbol{\beta} \\
(\mathbf{m} / \mathbf{s})\end{array}$ & Qs \\
\hline 1 & 20 & 34 & 16 \\
2 & 40 & 79 & 71 \\
Semi-espacio & ------ & 475 & 80 \\
\hline
\end{tabular}


Con el fin de verificar si este modelo es capaz de reproducir la respuesta de sitio observada (figuras 4 y 5), calculamos la función de transferencia mediante el método de Haskell-Thompson (Aki y Richards, 1980) considerando propagación vertical de ondas SH. En la figura 8 se muestran las comparaciones de la función de transferencia del modelo teórico -representada con una línea negra gruesa- con los cocientes H/V (línea continua delgada) y con los cocientes SSR de los componentes radial y transversal (líneas discontinuas). En esta figura se puede ver que la función de transferencia teórica tiene su frecuencia fundamental en $0.36 \mathrm{~Hz}$, mientras que las funciones empíricas la tienen en $0.37 \mathrm{~Hz}$; los niveles de amplificación estimados teóricamente corresponden más con las amplificaciones relativas al movimiento en la estación Estanzuela. Respecto a los armónicos superiores, el primer armónico de la función teórica corresponde en frecuencias con el definido por los cocientes $\mathrm{H} / \mathrm{V}$, mientras que el segundo corresponde con el estimado en los cocientes SSR y su amplitud es más parecida, aunque sigue siendo sobreestimada.

\section{CÁLCULO DE ESPECTROS DE RESPUESTA DE SITIO}

En la figura 9 se pueden ver dos bandas con seudoaceleraciones máximas: la primera entre 0 y $1 \mathrm{~s}$ de periodo, con una seudoaceleración normalizada cercana a $0.6 \mathrm{~g}$ y la segunda entre 2 y $3 \mathrm{~s}$ de periodo con valores de seudoaceleración de aproximadamente $0.8 \mathrm{~g}$. El intervalo entre 2 y $3 \mathrm{~s}$ de periodo corresponde con el periodo fundamental del sitio, mientras que el intervalo en periodos menores corresponde a un tercio del fundamental y está asociado con el primero modo superior.

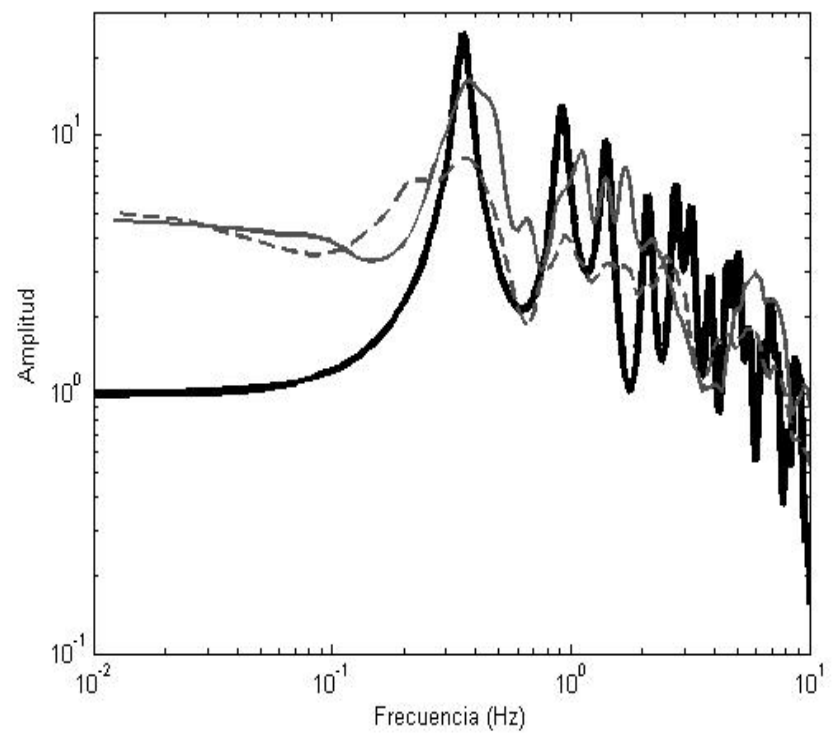

Figura 8. Comparación de las funciones de transferencia observadas (línea discontinua y continua delgada) y función de transferencia teórica (línea discontinua más gruesa).

Se puede ver que se tienen distintos comportamientos espectrales dependiendo del evento, ya que hay algunos que excitan los modos de propagación en los armónicos en bajos periodos (en la banda de 0 a $1 \mathrm{~s}$ de periodo); mientras que hay eventos cuya energía se propaga de manera más eficiente en periodos entre 2 y $3 \mathrm{~s}$. 


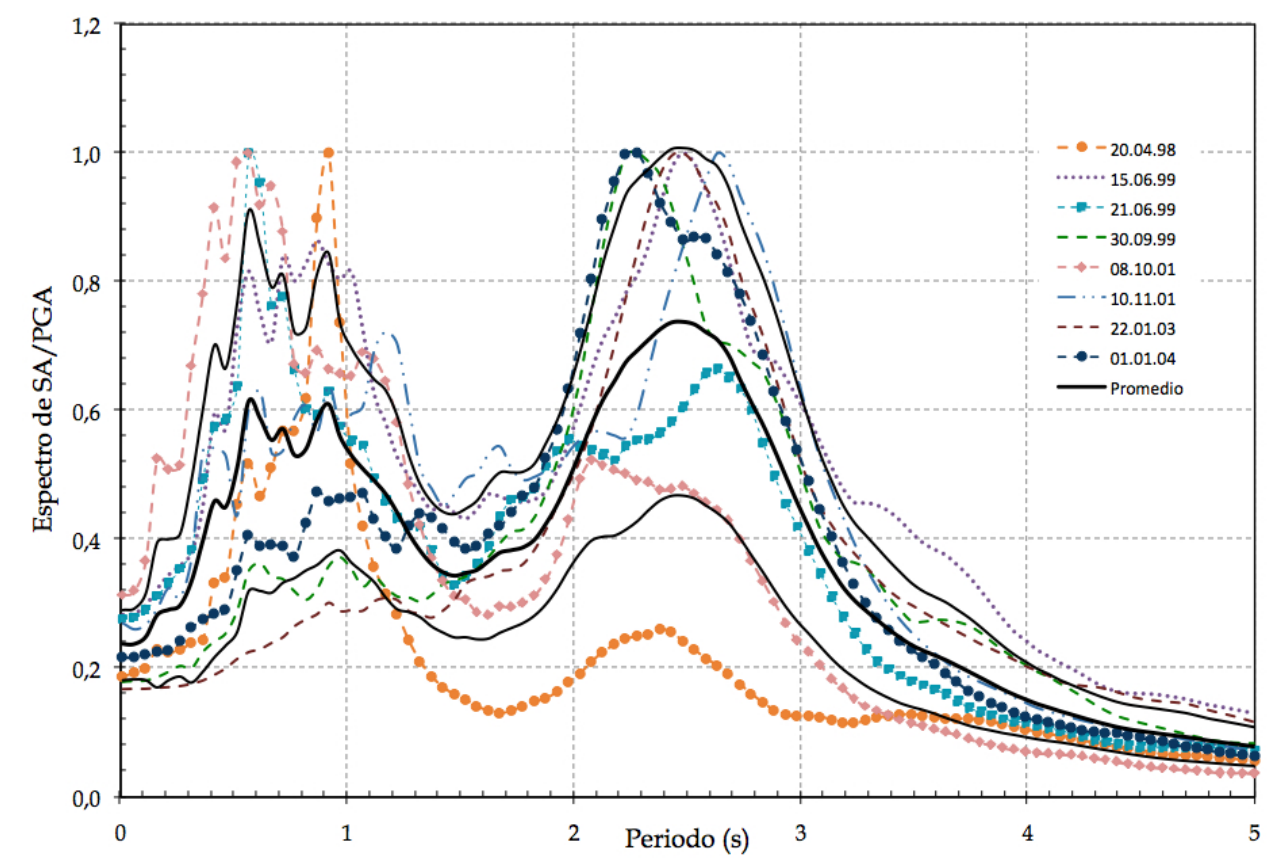

Figura 9. Espectros de seudoaceleración de sitio normalizados respecto de la PGA de cada evento, con 5\% de amortiguamiento. Los máximos se localizan entre 2 y 3 s de periodo.

\section{CONCLUSIONES}

La respuesta sísmica del sitio del arreglo Texcoco, obtenida a partir de cocientes espectrales $\mathrm{H} / \mathrm{V}$ y cocientes espectrales estándar, está dada por una frecuencia fundamental entre $0.37 \mathrm{~Hz}$. Los niveles de amplificación varían entre un factor de 8 , para los cocientes espectrales $\mathrm{H} / \mathrm{V}$, y cercanos a 17 para los cocientes espectrales estándar.

La obtención del modelo de velocidad de ondas de corte muestra que la estructura del subsuelo es simple y se trata de dos capas con velocidades de ondas de corte de 34 y $79 \mathrm{~m} / \mathrm{s}$, respectivamente, sobre un semiespacio a $38 \mathrm{~m}$ de profundidad con una velocidad $170 \mathrm{~m} / \mathrm{s}$. Utilizando el decaimiento exponencial de la amplitud del movimiento, obtuvimos valores del factor de atenuación Q que van de 34 a 29 en los primeros $40 \mathrm{~m}$ de profundidad. La confrontación de la función de transferencia observada con la obtenida del modelo de velocidad, y sus correspondientes valores de Q, muestra que la respuesta de sitio (la frecuencia fundamental) está dominada por la amplificación dinámica de las capas superficiales.

Finalmente, proponemos un espectro de respuesta de sitio para la zona de estudio. Los valores de seudoaceleración varían entre 0.6 g para periodos de 0 a 1 s y cercanos a 0.8 g para periodos entre 2 y $3 \mathrm{~s}$.

\section{AGRADECIMIENTOS}

Agradecemos la colaboración del Ing. Mauricio Ayala de la Coordinación de Instrumentación Sísmica del Instituto de Ingeniería de la UNAM, por su apoyo en la recolección de datos y el mantenimiento del arreglo TXCL, durante el periodo de funcionamiento. También agradecemos los comentarios y as críticas del Dr. Sergio Chávez Pérez que enriquecieron este trabajo, en cuanto a su forma y su contenido. 


\section{REFERENCIAS}

Aki, K y P G Richards (1980). Quantitative Seismology, W. H. Freeman.

Aguirre, J, H Mijares, E Briones-Ruiz, H Flores-Estrella y E Mares. (2001), "Aplicación n de observación de arreglos de microtremores a estudios geotécnicos mediante el método SPAC”, México, D.F.: Coordinación Editorial, Universidad Nacional Autónoma de México, Ciudad Universitaria.

Álvarez J, R E Chávez y C Lomnitz (1995). "Shallow SH-refraction survey on Mexico City mud: amplification by wedge effects”, First Break No. 13, 471-479.

Barker, J S, M Campillo, F J Sánchez-Sesma, D Jongmans y S K Singh (1996). "Analysis of wave propagation in the Valley of Mexico from a dense array of seismometers", Bull of the Seism Soc of Am, Vol. 86, pp. 1667-1680.

Beck, J L y J F Hall (1986). "Factors contributing to the catastrophe in Mexico City during the earthquake of September 19, 1985”, Geophys Res Lett, No. 13-6, 593-596.

Borcherdt, R D (1970). "Effects of local geology on ground motion near San Francisco Bay", Bull. Seism. Soc. Am., No. 60, 29-61.

Campillo M, J C Gariel, K Aki y F J Sánchez-Sesma (1989). "Destructive strong ground motion in Mexico City: source, path and site effects during the great 1985 Michoacán earthquake”, Bull. Seism. Soc. Am., No. 79, 1718-1735.

Cárdenas-Soto, M y F J Chávez-García (2007). “Aplicación de la interferometría sísmica para obtener la respuesta de edificios y depósitos de suelo ante movimientos fuertes”, XVI Congreso Nacional de Ingeniería Sísmica, Ixtapa-Zihuatanejo, Guerrero, México.

Cárdenas-Soto, M y F J Chávez-García (2007). "Earthquake ground motion in Mexico City: An analysis of data recorded at Roma Array”, Soil Dyn. and Earthq. Eng., No. 27, 475-486.

Cárdenas-Soto, M, F J Chávez-García y A Gusev (1997), "Regional amplification of ground motion in central Mexico. Results from coda magnitude data and preliminary modeling”, Journal of Seismology, No. 1, pp. 341-355.

Chávez-García, F J y M Cárdenas-Soto (2002). "The contribution of the built environment to the 'free-field' ground motion in Mexico City”, Soil Dyn. and Earthq. Eng., No. 22, 773-780.

Chávez-García, F J y L Salazar (2002). "Strong motion in Central Mexico: a model on data analysis and simple modeling”, Bull. Seism. Soc. Am., No. 92, 3087-3101.

Chávez-García, F J, J Ramos Martínez y E Romero-Jiménez (1995). "Surface-wave dispersion analysis in Mexico City”, Bull. Seism. Soc. Am., No. 85, 1116-1126.

Chávez-García F J y P Y Bard (1994). "Site effects in Mexico City eight years after the September 1985 Michoacán earthquake”, Soil Dyn Earthq Eng, No. 13, 229-247.

Chávez-García F J y P Y Bard (1993) “Gravity waves in Mexico City?” Part I and part II, Bull Seismol Soc Am, No. 83, 1637-1675.

Flores-Estrella, H (2004). "Método SPAC: Una alternativa para la estimación de modelos de velocidades en el Valle de México", Tesis de maestría, Posgrado en Ciencias de la Tierra, UNAM. 
Furumura, T y B L N Kennett (1998). "On the Nature of Regional Seismic Phases - III. The influence of crustal heterogeneity on the wavefield for subduction earthquakes: the 1985 Michoacán and the 1994 Copala, Guerrero, México earthquakes”, Geophys. J. Int., No. 135, 1060-1084.

Iida, M (1999), "Excitation of high-frequency surface waves with long duration in the Valley of Mexico”, J of Geophys Res, Vol. 104, 7329-7345.

Lay T y T C Wallace (1995). Modern Global Seismology. Academic Press.

Lermo, J y F J Chávez-García (1993). "Site effects evaluation using spectral ratios with only one station”, Bull. Seism. Soc. Am., No. 83, 1574-1594.

Lomnitz, C, J Flores, O Novaro, T H Seligman y R Esquivel (1999). “Seismic Coupling of Interface Modes in Sedimentary Basins: A Recipe for Disaster”, Bull. Seism. Soc. Am., No. 89-1, 1421.

Lomnitz, C (1988). Las causas probables de la catástrofe sísmica del 19 de septiembre de 1985. Ciencia y Desarrollo No. 14, 82.

Lomnitz, C (1990). “Mexico 1985, the case for gravity waves”. Geophys J Int No. 102, 569-572.

Kramer, S L (1996). Geotechnical Earthquake Engineering, Prentice Hall, 653 pp.

Malischewsky, A P G, C Lomnitz, F Wuttke y R Saragoni (2006). "Prograde Rayleigh-wave motion in the valley of Mexico", Geofísica Internacional, No. 45, 149-162.

Mayoral, J M, M P Romo y L Osorio (2008). "Seismic parameters characterization at Texcoco lake, Mexico”, Soil Dyn and Earthq Eng, No. 28, 507-521.

Ordaz, M y C Montoya-Dulché (2005). Programa Degtra A4.

Ordaz, M y S K Singh (1992). "Source spectra and spectral attenuation of seismic waves from Mexican earthquakes, and evidence of amplification in the hill zone of Mexico City", Bull. Seism. Soc. Am., No. 82, 24-43.

Ovando E, A Ossa y M P Romo (2007). "The sinking of Mexico City: its effect on soil properties and seismic response”, Soil Dyn Earthquake Eng, No. 27, 333-343.

Pacheco, F J y S K Singh. (1995), "Estimation of ground motions in the Valley of Mexico from normal-faulting, intermediate-depth earthquakes in the subducted Cocos plate", Earthq Spectra, No. 11, 233-247.

Pérez-Cruz, G (1988), “Estudio Sismológico de Reflexión del Subsuelo de la Ciudad de México”. Tesis de Maestría, Facultad de Ingeniería, UNAM, México.

Ramos-Martínez, J, F J Chávez García, E Romero Jiménez, J L Rodríguez-Zúñiga y J M Gómez González (1997), "Site effects in Mexico City; constraints from surface wave inversion of shallow refraction data”, J Appl Geophys, No. 36, 157-65.

RCDF (2004). "Reglamento de Construcciones para el Distrito Federal”, Administración Pública del Distrito Federal, Jefatura de Gobierno, Normas Técnicas Complementarias para el Diseño por Sismos, México.

Roullé, A y F J Chávez García (1997). "Comparison of three methods to study the wavefield in the Valley of Mexico using data from dense arrays”, J Appl Geophys, No. 36, 157-65.

Singh, S K, R Quaas, M Ordaz, F Mooser, D Almora, M Torres y R Vasquez (1995). "Is there truly a "hard” rock site in the Valley of Mexico?”, Geophys Res Lett No. 22, 481-484. 
Singh, S K y M Ordaz (1993). "On the origin of long coda observed in the lake-bed strong motion records of Mexico City”, Bull. Seism. Soc. Am., No. 83, 1298-1306.

Singh, S K, E Mena y R Castro (1988). "Some aspects of the source characteristics and ground motion amplifications in and near Mexico City from acceleration data of the September 1985 Michoacán, Mexico earthquakes”, Bull. Seism. Soc. Am., No. 78, 451-477.

Sneider, R y E Safak (2006). "Extracting the Building Response using Seismic Interferometry: Theory an Application to the Millikan Library in Pasadena, California”, Bull. Seism. Soc. Am., No. 96, 586-598.

Stephenson, W y C Lomnitz (2005). "Shear-Wave Velocity Profile at the Texcoco Strong-Motion Array Site, Valley of Mexico”; Geofísica Internacional, No. 44, 1, 3-10.

Stephenson, W, C Lomnitz y H. Flores-Estrella (2005). "Late resonant response at Texcoco, Valley of Mexico, during distant earthquakes”; Soil Dyn and Earthq Eng, No. 26, 791-79 